\title{
Higgs decay to goldstini and its observability at the LHC
}

\author{
Tao Liu, ${ }^{1}$ Lin Wang, ${ }^{1}$ and Jin Min Yang $^{2}$ \\ ${ }^{1}$ Institut für Theoretische Teilchenphysik, \\ Karlsruhe Institute of Technology (KIT), D-76128 Karlsruhe, Germany \\ ${ }^{2}$ State Key Laboratory of Theoretical Physics, \\ Institute of Theoretical Physics, Academia Sinica, Beijing 100190, China
}

\begin{abstract}
If supersymmetry is broken independently in multiple sectors with different scales, a number of goldstinos will be generated. One linear combination of these goldstinos is massless and eaten by the gravitino, while the orthogonal combinations acquire a tree level mass and become the physical states named goldstini $\left(G^{\prime}\right)$. Compared to the gravitino, such goldstini could couple more strongly to the visible fields and lead to some exotic phenomenology. In this note we first check the goldstini couplings in some GMSB models and find that the goldstini-photon-neutralino interaction may be very small while the goldstini-Z-neutralino and goldstini-Higgs-neutralino interactions may be sizable. This can induce a new decay mode for the Higgs boson: $h \rightarrow G^{\prime}+\chi \rightarrow Z+2 G^{\prime}$. Then in an effective model with conservative fixed parameters we study the observability of this decay channel at the LHC and find that it is not accessible at the finished $8 \mathrm{TeV}$ run $\left(25 \mathrm{fb}^{-1}\right)$ or $14 \mathrm{TeV}$ run with $100 \mathrm{fb}^{-1}$, but might be observed at the high luminosity LHC (14 TeV, 1000-3000 fb ${ }^{-1}$ ) if the systematics of the backgrounds can be well understood.
\end{abstract}

PACS numbers: 14.80.Da,14.80.Ly,12.60.Jv 


\section{INTRODUCTION}

Supersymmetry (SUSY) is a consistent extension of Poincaré symmetry in quantum field theory and can solve the hierarchy problem in the standard model due to the vanishing of quadratic divergence. However, in a realistic particle theory, SUSY must be broken. Usually it is assumed that SUSY breaking happens in a hidden sector and then is transmitted to low energy fields via certain mechanisms. This will generate a massless goldstino which acts as the longitude component of gravitino in supergravity theory. If SUSY breaking happens in only one sector, the interaction of the gravitino is given by [1, 2]

$$
\begin{aligned}
\mathcal{L}_{i n t} & =\frac{1}{F}\left(\partial_{\mu} G^{\alpha} J_{\alpha}^{\mu}+\text { h.c. }\right) \\
J^{\mu} & =\sigma^{\nu} \bar{\sigma}^{\mu} \psi D_{\nu} \phi^{*}-i \sigma^{\mu} \bar{\psi} F_{\phi}+i \frac{1}{2 \sqrt{2}} \sigma^{\nu} \bar{\sigma}^{\rho} \sigma^{\mu} \bar{\lambda} F_{\nu \rho}+\frac{1}{\sqrt{2}} \sigma^{\mu} \bar{\lambda} D
\end{aligned}
$$

where $\left(\phi, \psi, F_{\phi}\right)$ are the boson, fermi and $\theta^{2}$ components of a chiral superfield; $\left(\lambda, A_{\mu}, D\right)$ are the fermi, gauge and $\theta^{2} \bar{\theta}^{2}$ components of a vector superfield; $F_{\nu \rho}$ denotes the strength tensor of the gauge field $A_{\mu}$ and $D_{\mu}$ is the corresponding covariant derivative (throughout this paper we use the two component Weyl notation for the fields). The non-derivative couplings can be obtained after integrating by parts and using the equation of motion. From Eq.(1) we see that the gravitino does not play an important role in low energy phenomenology unless the SUSY breaking scale $F$ is sufficiently low.

However, if SUSY is broken in multiple sectors, some exotic phenomenology will be generated [3 11]. In such a scenario, each sector breaks SUSY independently at a scale $F_{i}$ and gives a goldstino $\eta_{i}$. One linear combination of $\eta_{i}$ is massless and eaten by the gravitino, while the orthogonal combinations named goldstini acquire a tree level mass $m_{G^{\prime}}=2 m_{3 / 2}$ in SUGRA [3] (possible loop corrections to the goldstini mass have also been considered [3, 8]). The true goldstino (longitudinal component of gravitino) has an interaction in Eq.(1) with $F=\sqrt{\sum_{i} F_{i}^{2}}$. Unconstrained by the supercurrent, the interaction of goldstini can be quite different and large enough to have intriguing phenomenology at colliders. For example, in the framework of gauge mediated SUSY breaking (GMSB), the goldstini can lead to some final states which are softer and more structured at colliders [11]. It can also serve as a dark matter candidate [6]. Additionally, as noted in [9], the goldstini may couple 'strongly' (much stronger than gravitino) with the lightest observable-sector supersymmetric particle (LOSP), which then may lead to some exotic decay channels for the Higgs boson, e.g., the Higgs may decay to the LOSP plus a goldstini.

Given the importance and urgency of Higgs physics, any possible exotic decays of the Higgs boson should be scrutinized. As is well known, a Higgs-like particle around $125 \mathrm{GeV}$ has been observed by ATLAS and CMS collaborations [12, 13], which motivated numerous 
theoretical studies, especially the enhanced diphoton decay has been intensively studied in various new physics models, such as the low energy SUSY [14], the little Higgs model [15], the two-Higgs-doublet model [16], the Higgs triplet model [17], the models with extra

dimensions [18] and other Higgs extensions [19]. In this note we focus on the effects of goldstini and examine the exotic Higgs decay mode $h \rightarrow \chi+G^{\prime}$ followed by $\chi \rightarrow Z+G^{\prime}$ under the condition $m_{Z}<m_{\chi}<m_{h}$ (here $\chi$ is the ordinary lightest neutralino which is assumed to be the LOSP, $G^{\prime}$ denotes the goldstini).

We will make a brief review on goldstini and explain why we focus on this channel in Section II. Then we take a model-independent way to study the Higgs decay $h \rightarrow \chi+G^{\prime}$ followed by $\chi \rightarrow Z+G^{\prime}$ at the LHC in Section III. Finally, we give our conclusion in Section IV.

\section{THEORETICAL MOTIVATIONS}

\section{A. A brief review on goldstini}

For simplicity we assume that there are only two sequestered sectors which break SUSY spontaneously. Following the arguments in [9], each of them can be parameterized in a non-linear way:

$$
X_{i}=\frac{\eta_{i}^{2}}{2 F_{i}}+\sqrt{2} \theta \eta_{i}+\theta^{2} F_{i}
$$

where $\eta$ is the so-called goldstino. Due to the non-renormalization theorem of superpotential, visible sector only obtains SUSY breaking information through non-trivial Kähler potential $K$ and gauge kinetic functions $f$. After integrating out hidden sector fields, $X_{i}$ couple to single species visible fields $\Phi$ as

$$
\begin{aligned}
K & =\Phi^{+} \Phi \sum_{i} \frac{m_{\phi, i}^{2}}{F_{i}^{2}} X_{i}^{+} X_{i} \\
f_{a b} & =\frac{1}{g_{a}^{2}} \delta_{a b}\left(1+\sum_{i} \frac{2 m_{a, i}}{F_{i}} X_{i}\right),
\end{aligned}
$$

where $g_{a}$ denote the gauge coupling constants, $m_{\phi, a}$ are respectively the soft masses for the scalar of the chiral superfield and gauginos. The trilinear and bilinear soft terms between multiple fields could also be constructed, but since they are subleading and model-dependent in the contribution to interactions between goldstino and visible sector, we do not consider them here. Substituting the expression of $X_{i}$ into the above formula gives the Lagrangian 
up to order $1 / F_{i}$ :

$$
\begin{aligned}
\mathcal{L}= & -\sum_{i} m_{\phi, i}^{2} \phi^{*} \phi+\sum_{i} \frac{m_{\phi, i}^{2}}{F_{i}} \eta_{i} \psi \phi^{*} \\
& -\frac{1}{2} \sum_{i} m_{a, i} \lambda^{a} \lambda^{a}-\sum_{i} \frac{i m_{a, i}}{\sqrt{2} F_{i}} \eta_{i} \sigma^{\mu \nu} \lambda^{a} F_{\mu \nu}^{a}+\sum_{i} \frac{m_{a, i}}{\sqrt{2} F_{i}} \eta_{i} \lambda^{a} D^{a} .
\end{aligned}
$$

The mass eigenstates can be obtained by a rotation of $\eta_{i}$ :

$$
G=\cos \theta \eta_{1}+\sin \theta \eta_{2}, \quad G^{\prime}=-\sin \theta \eta_{1}+\cos \theta \eta_{2}
$$

where $\theta$ is defined by $\tan \theta=F_{2} / F_{1}$. It is easy to see that the Weyl spinor $G$ is related to

SUSY breaking scale $F=\sqrt{F_{1}^{2}+F_{2}^{2}}$ while $G^{\prime}$ vanishes in the non-linear form. Then the interaction Lagrangian becomes

$$
\begin{aligned}
\mathcal{L}_{G} & =\frac{m_{\phi}^{2}}{F} G \psi \phi^{*}-\frac{i m_{a}}{\sqrt{2} F} G \sigma^{\mu \nu} \lambda^{a} F_{\mu \nu}^{a}+\frac{m_{a}}{F} G \lambda^{a} D^{a}, \\
\mathcal{L}_{G^{\prime}} & =\frac{\tilde{m}_{\phi}^{2}}{F} G^{\prime} \psi \phi^{*}-\frac{i \tilde{m}_{a}}{\sqrt{2} F} G^{\prime} \sigma^{\mu \nu} \lambda^{a} F_{\mu \nu}^{a}+\frac{\tilde{m}_{a}}{F} G^{\prime} \lambda^{a} D^{a},
\end{aligned}
$$

with the parameter $m$ and $\tilde{m}$ defined as

$$
m_{\phi / a}=m_{\phi / a, 1}+m_{\phi / a, 2}, \quad \tilde{m}_{\phi / a}=-m_{\phi / a, 1} \tan \theta+m_{\phi / a, 2} \cot \theta .
$$

$\mathcal{L}_{G}$ is just the non-derivative version of Eq.(1) and the couplings are proportional to the soft masses as expected. For goldstini, their interactions with visible fields could be enhanced as long as $\tilde{m}$ is larger than $m$. Note that if $D^{a}$ get a vacuum expectation value, there will be a mixing between $\lambda^{a}$ and $\eta_{i}$, which will give rise to some special interactions. In the scenario with approximate vanishing $\tilde{m}_{a}$ which we will discuss later, such a mixing can be safely neglected in the interaction of $G^{\prime}$.

The mass of goldstini is given at tree level by $m_{G^{\prime}}=2 m_{3 / 2}$ due to the intrinsic property of SUGRA. Additionally, there could be corrections to such a tree-level mass, which, however, are model-dependent [3, 8]. In our analysis we assume that both $m_{G}$ and $m_{G^{\prime}}$ are much smaller than the Higgs mass.

\section{B. Couplings between goldstini and Higgs boson}

Without the extra goldstini, the interaction of goldstino $(G)$ with the lightest neutralino $(\chi)$ is given by

$$
\mathcal{L}=\frac{y_{1}}{\sqrt{2} F} \chi G h+\frac{y_{2}}{2 \sqrt{2} F} \chi \sigma^{\mu \nu} G F_{\mu \nu}^{\gamma}+\frac{y_{3}}{2 \sqrt{2} F} \chi \sigma^{\mu \nu} G Z_{\mu \nu}+\frac{y_{4}}{\sqrt{2} F} \bar{\chi} \bar{\sigma}^{\mu} G Z_{\mu}+\text { h.c. }
$$


where $F_{\mu \nu}^{\gamma}$ is the photon field strength, $Z_{\mu}$ and $Z_{\mu \nu}$ are respectively the $Z$-boson field and its field strength. In the minimal supersymmetric standard model (MSSM), the above parameters $y_{1,2,3,4}$ are given by

$$
\begin{aligned}
y_{1}= & -N_{11}^{-1} m_{1} m_{Z} \sin \theta_{W} \sin (\alpha+\beta)+N_{21}^{-1} m_{2} m_{Z} \cos \theta_{W} \sin (\alpha+\beta) \\
& +N_{31}^{-1}\left(B_{\mu} \cos \alpha-m_{H_{d}}^{2} \sin \alpha\right)+N_{41}^{-1}\left(-B_{\mu} \sin \alpha+m_{H_{u}}^{2} \cos \alpha\right), \\
y_{2}= & -2 i m_{\chi}\left(N_{11}^{*} \cos \theta_{W}+N_{12}^{*} \sin \theta_{W}\right), \\
y_{3}= & -2 i m_{\chi}\left(-N_{11}^{*} \sin \theta_{W}+N_{12}^{*} \cos \theta_{W}\right)-2 i m_{Z}\left(N_{13}^{*} \cos \beta-N_{14}^{*} \sin \beta\right), \\
y_{4}= & i m_{Z}^{2}\left(-N_{11}^{*} \sin \theta_{W}+N_{12}^{*} \cos \theta_{W}\right)+i m_{Z} m_{\chi}\left(N_{13}^{*} \cos \beta-N_{14}^{*} \sin \beta\right),
\end{aligned}
$$

where $N_{1 i}$ denote the mixing between the lightest neutralino and the gauginos or higgsinos. We see that unless the SUSY breaking scale $F$ is small enough, such interactions of goldstino could hardly affect the $125 \mathrm{GeV}$ Higgs.

Naively speaking, the interactions of the goldstini can be obtained from the above expression with the goldstino $G$ replaced by the goldstini $G^{\prime}$ and each soft mass replaced by its corresponding $\tilde{m}$. Then it is clear that for $\tilde{m} \gg m$ the interactions of the goldstini can be significantly stronger than the goldstino. However, there are some subtle differences which deserve attention.

Next, we scrutinize the concrete low energy interactions of the goldstini. The first one is its interaction with Higgs and neutralino: $\frac{\tilde{y}_{1}}{\sqrt{2} F} \chi G^{\prime} h$. From Eq.(8) we can obtain the coefficient of this interaction:

$$
\begin{aligned}
\tilde{y}_{1}= & -N_{11}^{-1} \tilde{m}_{1} m_{Z} \sin \theta_{W} \sin (\alpha+\beta)+N_{21}^{-1} \tilde{m}_{2} m_{Z} \cos \theta_{W} \sin (\alpha+\beta) \\
& +N_{31}^{-1}\left(\tilde{B}_{\mu} \cos \alpha-\tilde{m}_{H_{d}}^{2} \sin \alpha\right)+N_{41}^{-1}\left(-\tilde{B}_{\mu} \sin \alpha+\tilde{m}_{H_{u}}^{2} \cos \alpha\right) .
\end{aligned}
$$

Similarly, the interaction with photon and neutralino takes a form of $\frac{\tilde{y}_{2}}{2 \sqrt{2} F} \chi \sigma^{\mu \nu} G^{\prime} F_{\mu \nu}^{\gamma}$ with

$$
\tilde{y}_{2}=-2 i m_{\chi}\left[\frac{\tilde{m}_{1}}{m_{1}} N_{11}^{*} \cos \theta_{W}+\frac{\tilde{m}_{2}}{m_{2}} N_{12}^{*} \sin \theta_{W}\right] .
$$

The interaction with $Z$-boson and neutralino is somewhat complicated. But based on the experience obtained above, some hints could be obtained. From the expression of $y_{3 / 4}$, it can be easily found that the $Z$-boson can be divided into two parts: one is its transverse component proportion to $\left(-N_{11}^{*} \sin \theta_{W}+N_{12}^{*} \cos \theta_{W}\right)$, and the other is the longitude component proportional to $\left(N_{13}^{*} \cos \beta-N_{14}^{*} \sin \beta\right)$. The interaction of goldstini with the transverse part of the $Z$-boson is almost the same as photon because they come from the same origin $\frac{i m_{a, i}}{\sqrt{2} F_{i}} \eta_{i} \sigma^{\mu \nu} \lambda^{a} F_{\mu \nu}^{a}$. After multiplying a factor $\tilde{m}_{1} / m_{1}$ to $N_{11}^{*} \cos \theta_{W}$ and a factor $\tilde{m}_{2} / m_{2}$ to $N_{12}^{*} \sin \theta_{W}$ in Eqs.(13,14), we can get the coefficient of this transverse coupling, which is proportional to $\tilde{m}_{1}$ or $\tilde{m}_{2}$. For the longitude component of the $Z$ boson, since it has some 
relations with the tilted mass parameters of the Higgs, such as $\tilde{m}_{h_{u, d}}$ and $\tilde{\mu}$, we can not get the same simple result. In [11] a free factor $K_{Z_{L}}$ is introduced to connect the longitudinal interaction between $G$ and $G^{\prime}$. Although we do not know the exact formula for $K_{Z_{L}}$, it must be a function of $\tilde{m}_{\phi} / m_{\phi}$ from naive arguments and dimensional analysis (here $m_{\phi}$ denotes the soft Higgs parameters). The details of the coupling between $Z$-boson and goldstini can be found in the appendix of [9].

From the above analysis we see that the $G^{\prime}-\gamma-\chi$ and $G^{\prime}-Z-\chi$ (transverse $Z$ ) couplings are both proportional to $\tilde{m}_{a}$ (tilted gaugino masses) while the $G^{\prime}-Z-\chi$ (longitudinal $Z$ ) and $G^{\prime}-h-\chi$ couplings are proportional to $\tilde{m}_{\phi}$. So, if $\tilde{m}_{a}$ are very small while $\tilde{m}_{\phi} / m_{\phi}$ is much larger than one, we can get a new scenario in which the Higgs decay $h \rightarrow \chi G^{\prime} \rightarrow Z G^{\prime} G^{\prime}$ is more sizable than $h \rightarrow \chi G^{\prime} \rightarrow \gamma G^{\prime} G^{\prime}$. Note that from the viewpoint of model-building, this scenario could be easily realized in a two-sector messenger with $F_{1} \gg F_{2}$. If the second sector preserves $R$-symmetry, it will give no contribution to the gaugino masses. Besides, in

some concrete GMSB models, especially the direct gauge mediation [21], the gaugino masses are usually suppressed by a factor $F_{i}^{2} / M_{i}^{4}$ due to the vacuum structure of superfields. So it is common to have approximately vanishing $\tilde{m}_{a}$. Additionally, in the case of stimulated SUSY breaking [22], it is quite natural to split $F_{1}$ and $F_{2}$.

\section{HIGGS DECAY TO GOLDSTINI AT THE LHC}

From the analysis in the preceding section, we see that in some GMSB models the goldstini couplings $G^{\prime}-h-\chi$ and $G^{\prime}-Z-\chi$ can be sizable while the coupling $G^{\prime}-\gamma-\chi$ can be much suppressed. This scenario can lead to the Higgs decay $h \rightarrow \chi G^{\prime} \rightarrow Z G^{\prime} G^{\prime}$ which will be studied in this section.

\section{A. The rate of Higgs decay to goldstini}

We take an effective way to study the Higgs decay $h \rightarrow \chi G^{\prime} \rightarrow Z G^{\prime} G^{\prime}$. The effective Lagrangian is given by

$$
\mathcal{L}_{e f f}=\frac{m^{2}}{F}\left[g_{h \chi} h \chi G^{\prime}+g_{\chi Z} \bar{G}^{\prime} \bar{\sigma}^{\mu} Z_{\mu} \chi+\text { h.c. }\right]
$$

Here a mass parameter $m$ is introduced to make the couplings $g_{h \chi}$ and $g_{\chi} z$ dimensionless and the Lagrangian takes the similar form as in [23] which studied the signal of mono-photon plus missing energy from the Higgs decay. Note that for $m_{\chi}>m_{h}$, the lightest neutralino can decay to the Higgs boson which may lead to many boosted Higgs bosons at the LHC [24]. 
As shown in Fig. 1, the decay branching ratio of $h \rightarrow Z G^{\prime} G^{\prime}$ is very small for an off-shell neutralino $\left(h \rightarrow \chi^{*} G^{\prime} \rightarrow Z G^{\prime} G^{\prime}\right)$ but can be sizable for an on-shell neutralino $\left(h \rightarrow \chi G^{\prime}\right.$ followed by $\chi \rightarrow Z G^{\prime}$ ). The partial decay width of $h \rightarrow \chi G^{\prime}$ is given by

$$
\Gamma\left(h \rightarrow \chi G^{\prime}\right)=\frac{m_{h}}{8 \pi} \frac{g_{h \chi}^{2} m^{4}}{F^{2}}\left(1-\frac{m_{\chi}^{2}}{m_{h}^{2}}\right)^{2} .
$$
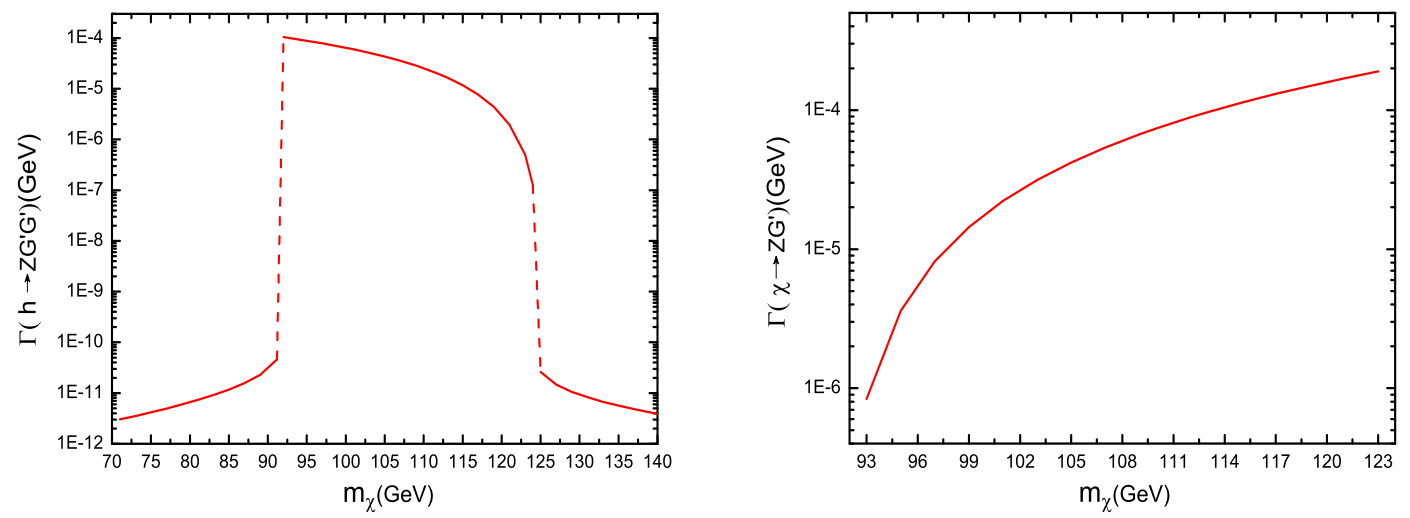

FIG. 1: The partial widths of $h \rightarrow Z G^{\prime} G^{\prime}$ and $\chi \rightarrow Z G^{\prime}$ versus the neutralino mass. Here we choose $m=0.1 \sqrt{F}$ with $\sqrt{F}=1.5 \mathrm{TeV}$, and $g_{h \chi}=g_{\chi Z}=1$.

Note that there are already some constraints on the whole SUSY breaking scale $\sqrt{F}$ through the interaction of gravitino (usually photon is also involved). The current lower bound is around $300 \mathrm{GeV}$ [25, 26] and it is expected that the LHC could push it to about 1.6 $\mathrm{TeV}$ [27]. The most sensitive process to $\sqrt{F}$ is the decay channel $h \rightarrow \chi G^{\prime}$ whose branching ratio is proportional to $1 / F$. In our calculation we fix $\sqrt{F}=1.5 \mathrm{TeV}$ and $m=0.1 \sqrt{F}$, and assume all the dimensionless couplings to be unity. Note that here we only take a conservative value for the parameter $m$. Since it is proportional to the tilted soft mass, as discussed in the preceding section, it can be much larger.

About the decay of the neutralino $\chi$, it may have some other modes, e.g., decay to SM particles if R-parity is violated or decay to a light $U(1)_{X}$ gaugino [28]. In our calculation we assume that $\chi$ only decays to $Z G^{\prime}$. If there are other decay modes, the corresponding signal should be multiplied by the branching ratio $\operatorname{Br}\left(\chi \rightarrow Z G^{\prime}\right)$. Additionally, the decay length of the neutralino with energy $\mathrm{E}$ is approximately $\Gamma^{-1} \sqrt{\left(E^{2}-m_{\chi}^{2}\right) / m_{\chi}^{2}} \sim 10^{-10} \mathrm{~cm}$, so the neutralino will decay inside the detector.

\section{B. Signal and background}

With the goldstini couplings in Eq.(17), the final state $Z G^{\prime} G^{\prime}$ can come from three processes at the LHC, as shown in Fig. 2, Generally, the processes (a) and (b) will generate 
$Z$-boson with low transverse momentum, while (c) with $t$-channel squark exchange may generate a $Z$-boson with large transverse momentum [27]. Additionally, heavier squarks which are consistent with the result of LHC will suppress the $t$-channel contributions, so we will not consider the process (c).

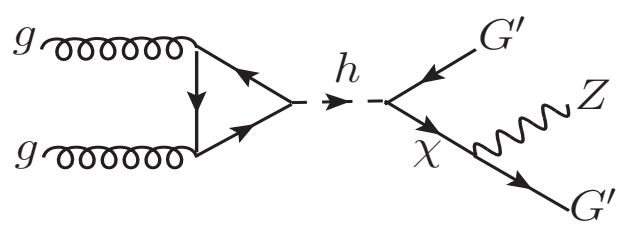

(a)

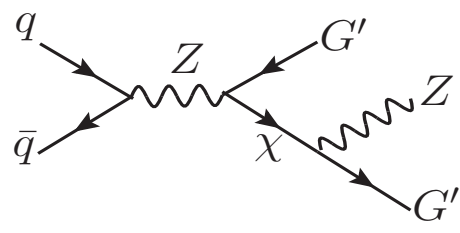

(b)

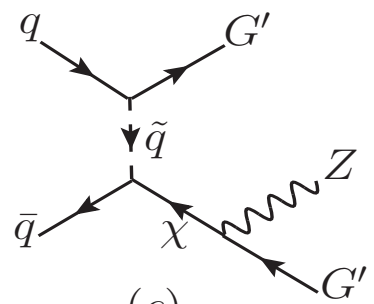

(c)

FIG. 2: Feynman diagrams for the production of $Z G^{\prime} G^{\prime}$ at the LHC induced by the goldstini couplings in Eq. (17).

We require the $Z$-boson produced in association with goldstini to decay to electrons or muons. Therefore, the signal is a pair of electrons or muons and low missing transverse momentum, i.e., $p p \rightarrow Z+E_{t} \rightarrow l^{+} l^{-}+E_{t}(l=e, \mu)$. Some studies about such mono-Z process have been performed in [29 31], where the mono-Z has a large transverse momentum. In our signal, the $Z$-boson has small transverse momentum because it comes from the decay of the neutralino.

Note that the direct neutralino pair production $p p \rightarrow \chi \chi$ followed by the decay $\chi \rightarrow Z G^{\prime}$ with one $Z$-boson decay to leptons and the other to neutrinos can also give the final state $l^{+} l^{-}+E_{t}$. We checked that the rate of this process is much smaller than the $s$-channel processes shown in Fig.2. So we can neglect it safely.

For the $s$-channel process shown in Fig.2(a), the Higgs boson can be produced on-shell and then decay to the $Z$-boson plus missing energy. For such a production we can get the high order corrections by multiplying a $k$-factor. At the LHC, the leading order Higgs boson production in the SM is from the gluon-gluon fusion. We calculate the Higgs boson production cross section at $\sqrt{s}=14 \mathrm{TeV}$ with CTEQ6M parton distribution functions. We set the renormalization scale $\mu_{R}$ and factorization scale $\mu_{F}$ at the Higgs mass of $125 \mathrm{GeV}$. The other relevant parameters are set as

$$
m_{t}=173.3 \mathrm{GeV}, \quad m_{b}=4.67 \mathrm{GeV}, \quad m_{Z}=91.188 \mathrm{GeV}, \quad m_{W}=80.4 \mathrm{GeV}
$$

We consider the higher order corrections and follow [23] to get $\sigma_{h}^{N N L O}=49.99 \mathrm{pb}$ at the next-to-next-to leading order with next-to-next-to leading logarithm resummation. For the $Z$-boson $s$-channel process shown in Fig.2(b), the neutralino can be produced on-shell and 
then decay to $Z$-boson and $G^{\prime}$. So far no high order corrections have been calculated for this process and we calculate this production at the leading order.

The main SM backgrounds are

$$
\begin{aligned}
& p p \rightarrow Z Z \rightarrow l^{+} l^{-} \nu \bar{\nu}, \\
& p p \rightarrow W^{+} W^{-} \rightarrow l^{+} \nu l^{-} \bar{\nu}, \\
& p p \rightarrow Z j \rightarrow l^{+} l^{-} j, \\
& p p \rightarrow t \bar{t} \rightarrow b \bar{b} l^{+} l^{-} \nu \bar{\nu} \\
& p p \rightarrow Z W^{ \pm} \rightarrow l^{+} l^{-} l^{ \pm} \nu .
\end{aligned}
$$

Obviously, the first two processes are our irreducible backgrounds. Because they proceed through the electroweak interaction, their cross sections are expected to be relatively small at the LHC. The third process can mimic our signal when the jet is missing detection, which is detector-dependent. For the forth process to mimic our signal, the two $b$-jets must be missing, which is less likely. However, since the $t \bar{t}$ production rate is large at the LHC, we cannot ignore it. For the last process, one lepton must be missing. Since its cross section is much smaller than the third process, we neglect it in our calculation.

In our calculation we use MadGraph5 [20] for both the signal and backgrounds. For $Z+$ jet process, we carry out parton shower by Pythia [32] and match the matrix element with parton shower in the $k_{T}$-jet MLM scheme [33]. We perform a fast detector simulation by using Delphes [34] and use the anti- $k_{t}$ algorithm [35] with the radius parameter $\Delta R=0.6$ to cluster jets.

\section{Numerical results}

Before giving the numerical result on the signal and backgrounds, we summarize our selection criteria on the final state:

$$
\begin{gathered}
P_{T}^{l}>20 \mathrm{GeV}, \quad E_{T}>20 \mathrm{GeV}, \quad\left|\eta_{l}\right|<2.5, \quad \Delta R \equiv \sqrt{\Delta \eta^{2}+\Delta \phi^{2}}>0.4, \\
\text { no jet with } P_{T}^{j}>20 \mathrm{GeV} \text { and }\left|\eta_{j}\right|<4.0
\end{gathered}
$$

where the separation $\Delta R$ is for the leptons in the final state with $\Delta \eta$ being the pseudorapidity difference and $\Delta \phi$ being the azimuthal angle difference. The jet veto and the lower cut on the missing energy can greatly suppress the $Z+$ jet and $t \bar{t}$ backgrounds. We further require the invariant mass of the dileptons to peak at $Z$-boson mass and apply an upper cut of $30 \mathrm{GeV}$ on the missing energy (for the main signal process $p p \rightarrow h \rightarrow Z+E_{t}$, the missing transverse momentum is below about $30 \mathrm{GeV}$ ). 
TABLE I: The number of signal and background events for the LHC with $\sqrt{s}=14 \mathrm{TeV}$ and 10 $\mathrm{fb}^{-1}$ of integrated luminosity.

\begin{tabular}{||c||c|c|c|c|c||c|c|c||}
\hline \hline \multirow{2}{||c||}{$\begin{array}{c}\sqrt{s}=14 \mathrm{TeV} \\
\left(10 f b^{-1}\right)\end{array}$} & \multicolumn{5}{c||}{ Background (B) } & \multicolumn{3}{c||}{ Signal (S) } \\
\cline { 2 - 11 } & $Z Z$ & $W^{+} W^{-}$ & $t \bar{t}$ & $Z j$ & $B_{t o t}$ & $S_{h}$ & $S_{Z}$ & $S_{\text {tot }}$ \\
\hline selection criteria & 964 & 12598 & 94 & 4812 & 18468 & 31 & 1.9 & 32.9 \\
\hline$\left|m_{l l}-m_{Z}\right|<5 \mathrm{GeV}$ & 834 & 900 & 35 & 3609 & 5378 & 25 & 1.7 & 26.7 \\
\hline$E_{T}<30 \mathrm{GeV}$ & 133 & 214 & 0 & 3609 & 3956 & 25 & 0.5 & 25.5 \\
\hline \hline
\end{tabular}

In Table I we present the number of events for the LHC with $\sqrt{s}=14 \mathrm{TeV}$ and $10 f b^{-1}$ of integrated luminosity. In our calculation of the signal, the mass parameters in Eq. (17) are fixed as $m_{\chi}=110 \mathrm{GeV}, m_{G^{\prime}}=0$ (the goldstini is much lighter than the Higgs, so we set it to zero for simplicity), $m=0.1 \sqrt{F}, \sqrt{F}=1.5 \mathrm{TeV}$, while all the dimensionless couplings are fixed to unity. From this table we see that the signal is overwhelmed by the backgrounds. As expected, the cut on the invariant dilepton mass $\left|m_{l l}-m_{Z}\right|<5 \mathrm{GeV}$ can suppress the $W^{+} W^{-}$and $t \bar{t}$ backgrounds efficiently. The upper limit cut on the missing transverse momentum can suppress the $Z Z, W^{+} W^{-}$and $t \bar{t}$ processes significantly. But the $Z+$ jet process cannot be suppressed by this cut because here the missing jet has a low transverse momentum [30].

Finally, we show in Table II the signal significance for different integrated luminosity. Since the contribution from the $s$-channel $Z$-boson process is much smaller than the Higgs process (as shown in Table I), in Table II we only consider the Higgs process so that the result could be interpreted as the product of the Higgs production rate, the Higgs decay branching ratio and the neutralino decay branching ratio.

Note that in Table II we only show the statistical significance. Since the ratio of signal to background is quite small (around 0.6 percent), we need a quite high luminosity to get a good statistic significance. However, the detection of such a rare process also needs a good understanding of the systematical uncertainty of the backgrounds, which is always a challenging job for hadron colliders like the LHC. These backgrounds (especially the Z+jets) are also the backgrounds for the Higgs signal $Z Z^{*}$ and so far at the LHC their systematical uncertainty is at a few percent level, as shown in Table 6 in [36]. In the future high luminosity $14 \mathrm{TeV}$ LHC, the systematics of such backgrounds need to be further improved for precision measurement of the Higgs property and the probe of new physics. Theoretically, we can have a larger ratio of signal to background if we enlarge the effective mass parameter $m$ (in our above analysis we took a conservative value of $0.1 \sqrt{F}$ for illustration). 
TABLE II: Same as Table I, but showing the event number of the signal $p p \rightarrow h \rightarrow \chi G^{\prime} \rightarrow$ $Z G^{\prime} G^{\prime} \rightarrow l^{+} l^{-}+Z_{t}$ and its statistical significance for the LHC with $\sqrt{S}=14 \mathrm{TeV}$ and different luminosity.

\begin{tabular}{||c|c|c|c|c|c|c||}
\hline \hline$\sqrt{S}=14 \mathrm{TeV}$ & $100 \mathrm{fb}^{-1}$ & $500 \mathrm{fb}^{-1}$ & $800 \mathrm{fb}^{-1}$ & $1000 \mathrm{fb}^{-1}$ & $2000 \mathrm{fb}^{-1}$ & $3000 \mathrm{fb}^{-1}$ \\
\hline$S_{[\text {selection criteria }]}$ & 310 & 1550 & 2480 & 3100 & 6200 & 9300 \\
\hline$S_{[\text {passing all cut }]}$ & 250 & 1250 & 2000 & 2500 & 5000 & 7500 \\
\hline$S / \sqrt{S+B}$ & 1.3 & 2.8 & 3.5 & 4.0 & 5.6 & 6.8 \\
\hline \hline
\end{tabular}

\section{CONCLUSION}

Compared with the gravitino, the goldstini can couple more strongly to the visible fields and thus induce some interesting phenomenology. In this note we considered the effects of the goldstini on the Higgs phenomenology. We found that in some GMSB models the goldstini has approximately vanishing interaction with photon and the lightest neutralino, but the corresponding coupling with the $Z$-boson is sizable. This could induce the mono- $Z$ decay of the Higgs boson $\left(h \rightarrow G^{\prime}+\chi \rightarrow Z+2 G^{\prime}\right)$ which signals $Z$-boson and $E_{t}$ at the LHC. Then in an effective model with conservative fixed parameters we studied the observability of this decay at the LHC. From Monte Carlo simulation of the signal and backgrounds, we found that it is not accessible at the finished $8 \mathrm{TeV}$ run $\left(25 \mathrm{fb}^{-1}\right)$ or $14 \mathrm{TeV}$ run with $100 \mathrm{fb}^{-1}$, but might be observed at the high luminosity LHC $\left(14 \mathrm{TeV}, 1000-3000 \mathrm{fb}^{-1}\right)$ if the systematics of the backgrounds can be well understood. Although at the LHC it is so challenging to detect this exotic decay channel of the Higgs boson, it is worth hunting because such a scenario may naturally exist in GMSB with multi SUSY breaking sectors.

\section{Acknowledgments}

We would like to thank Olivier Mattelaer for his great help on MadGraph and Lei Wu for useful discussions. Lin Wang acknowledges Prof. Johann H. Kühn and Prof. Matthias Steinhauser for their warm hospitality. This work is supported by DFG through SFB/TR 9 "Computational Particle Physics" and by the National Natural Science Foundation of China under grant Nos. 11275245, 10821504 and 11135003.

[1] P. Fayet, Phys. Lett. B 70, 461 (1977).

[2] H. K. Dreiner, H. E. Haber and S. P. Martin, Phys. Rept. 494, 1 (2010). 
[3] C. Cheung, Y. Nomura and J. Thaler, JHEP 1003, 073 (2010).

[4] C. Cheung, J. Mardon, Y. Nomura and J. Thaler, JHEP 1007, 035 (2010).

[5] N. Craig, J. March-Russell and M. McCullough, JHEP 1010, 095 (2010).

[6] H. -C. Cheng, W. -C. Huang, I. Low and A. Menon, JHEP 1103, 019 (2011).

[7] K. Mawatari and Y. Takaesu, Eur. Phys. J. C 71, 1640 (2011).

[8] R. Argurio, Z. Komargodski and A. Mariotti, Phys. Rev. Lett. 107, 061601 (2011).

[9] J. Thaler and Z. Thomas, JHEP 1107, 060 (2011).

[10] C. Cheung, F. D'Eramo and J. Thaler, JHEP 1108, 115 (2011).

[11] R. Argurio et al., JHEP 1206, 096 (2012).

[12] G. Aad et al. [ATLAS Collaboration], Phys. Lett. B 716, 1 (2012).

[13] S. Chatrchyan et al. [CMS Collaboration], Phys. Lett. B 716, 30 (2012).

[14] M. Carena et al. JHEP 1203, 014 (2012); JHEP 1207, 175 (2012); J. Cao et al., JHEP 1203, 086 (2012); JHEP 1210, 079 (2012); Phys. Lett. B 710, 665 (2012); Phys. Lett. B 703, 462 (2011); arXiv:1301.4641; U. Ellwanger, JHEP 1203, 044 (2012); U. Ellwanger, C. Hugonie, arXiv:1203.5048; A. Arbey et al., JHEP 1209, 107 (2012); K. Hagiwara, J. S. Lee, J. Nakamura, JHEP 1210, 002 (2012); R. Benbrik et al., arXiv:1207.1096; N. Christensen, T. Han, S. Su, arXiv:1203.3207; T. Cheng, arXiv:1207.6392; B. Kyae, J.-C. Park, arXiv:1207.3126; H. An, T. Liu, L.-T. Wang, arXiv:1207.2473; J. F. Gunion, Y. Jiang and S. Kraml, arXiv:1207.1545; J. Ke et al., arXiv:1207.0990; G. Belanger et al., arXiv:1208.4952, M. Drees, arXiv:1210.6507; S. F. King et al., arXiv:1211.5074; K. Choi et al., arXiv:1211.0875; M. Berg et al., arXiv:1212.5009; L. Aparicio et al., arXiv:1212.4808; C. Balazs, S. K. Gupta, arXiv:1212.1708; K. Cheung, C.-T. Lu, T.-C. Yuan, arXiv:1212.1288; A. Chakraborty et al., arXiv:1301.2745;

[15] J. Reuter, M. Tonini, arXiv:1212.5930; X.-F. Han et al., arXiv:1301.0090; L. Wang J. M. Yang, Phys. Rev. D 84, 075024 (2011); Phys. Rev. D 79, 055013 (2009).

[16] C. Haluch, R. Matheus, Phys. Rev. D 85, 095016 (2012); X.-G. He, B. Ren, J. Tandean, Phys. Rev. D 85, 093019 (2012); A. Arhrib, R. Benbrik, C.-H. Chen, arXiv:1205.5536; E. Cervero and J.-M. Gerard, arXiv:1202.1973; L. Wang, X.-F. Han, JHEP 1205, 088 (2012); A. Drozd et al., arXiv:1211.3580; S. Chang et al., arXiv:1210.3439; N. Chen, H.-J. He, JHEP 1204, 062 (2012); T. Abe, N. Chen, H.-J. He, arXiv:1207.4103; C. Han et al., arXiv:1212.6728.

[17] A. G. Akeroyd, S. Moretti, Phys. Rev. D 86, 035015 (2012); A. Arhrib et al., JHEP 1204, 136 (2012); L. Wang, X.-F. Han, Phys. Rev. D 86, 095007 (2012); Phys. Rev. D 87, 015015 (2013).

[18] G. Blanger et al., arXiv:1201.5582; K. Cheung, T.-C. Yuan, Phys. Rev. Lett. 108, 141602 (2012). 
[19] Y. Cai, W. Chao, S. Yang, arXiv:1208.3949; S. Chang et al., arXiv:1207.0493; J. Berger, J. Hubisz, M. Perelstein, JHEP 1207, 016 (2012); S. Dawson, E. Furlan, Phys. Rev. D 86, 015021 (2012); S. Dawson, E. Furlan, I. Lewis, arXiv:1210.6663; M. Chala, arXiv:1210.6208; C. -S. Chen et al., arXiv:1301.4694.

[20] J. Alwall et al., JHEP 1106, 128 (2011).

[21] Z. Komargodski and D. Shih, JHEP 0904, 093 (2009).

[22] M. McCullough, Phys. Rev. D 82, 115016 (2010).

[23] C. Petersson, A. Romagnoni and R. Torre, JHEP 1210, 016 (2012).

[24] K. Howe and P. Saraswat, JHEP 1210, 065 (2012).

[25] D. Acosta et al. [CDF Collaboration], Phys. Rev. Lett. 89, 281801 (2002).

[26] P. Achard et al. [L3 Collaboration], Phys. Lett. B 587, 16 (2004).

[27] A. Brignole, F. Feruglio, M. L. Mangano and F. Zwirner, Nucl. Phys. B 526, 136 (1998).

[28] M. Baryakhtar, N. Craig and K. Van Tilburg, JHEP 1207, 164 (2012).

[29] F. J. Petriello, S. Quackenbush and K. M. Zurek, Phys. Rev. D 77, 115020 (2008).

[30] N. F. Bell et al., Phys. Rev. D 86, 096011 (2012).

[31] L. M. Carpenter et al., arXiv:1212.3352.

[32] T. Sjostrand, L. Lonnblad, S. Mrenna and P. Z. Skands, hep-ph/0308153.

[33] J. Alwall et al., Eur. Phys. J. C 53, 473 (2008)

[34] S. Ovyn, X. Rouby and V. Lemaitre, arXiv:0903.2225 [hep-ph].

[35] M. Cacciari, G. P. Salam and G. Soyez, JHEP 0804, 063 (2008)

[36] The ATLAS Collaboration, JHEP03, 128 (2013). 